\title{
Esterification Prevents Induction of the Mitochondrial Permeability Transition by $N$-Acetyl Perfluorooctane Sulfonamides
}

\author{
Timothy M. O’Brien, ${ }^{*}{ }^{\dagger}$ Robert M. Carlson, ${ }^{\ddagger}$ Paulo J. Oliveira, ${ }^{\S}$ and Kendall B. Wallace ${ }^{\dagger}$ \\ Department of Biochemistry and Molecular Biology, Toxicology Graduate Program, University of Minnesota, \\ Medical School, 1035 University Drive, Duluth, Minnesota 55812, Department of Chemistry, University of \\ Minnesota, 1035 University Drive, Duluth, Minnesota 55812, and Center for Neurosciences and Cell Biology, \\ Department of Zoology, University of Coimbra, Coimbra, Portugal
}

Received June 19, 2006

\begin{abstract}
$\mathrm{N}$-Alkyl perfluorooctane sulfonamides have been widely used as surfactants on fabrics and papers, fire retardants, and anticorrosion agents, among many other commercial applications. The broad use, global distribution, and environmental persistence of these compounds has generated considerable interest regarding potentially toxic effects. We have previously reported that perfluorooctanesulfonamidoacetate (FOSAA) and $N$-ethylperfluorooctanesulfonamidoacetate ( $N$-EtFOSAA) induce the mitochondrial permeability transition (MPT) in vitro, resulting in cytochrome $c$ release, inhibition of respiration, and generation of reactive oxygen species. By synthesizing the corresponding methyl esters of FOSAA and $N$-EtFOSAA (methyl perlfuorinated sulfonamide acetates), we tested the hypothesis that the $N$-acetate moiety of FOSAA and $N$-EtFOSAA is the functional group responsible for induction of the MPT. Swelling of freshly isolated liver mitochondria from Sprague-Dawley rats was monitored spectrophotometrically and membrane potential $(\Delta \Psi)$ was measured using a tetraphenylphosphonium-selective $\left(\mathrm{TPP}^{+}\right)$electrode. In the presence of calcium, $40 \mu \mathrm{M}$ FOSAA and $7 \mu \mathrm{M} N$-EtFOSAA each induced mitochondrial swelling and a biphasic depolarization of membrane potential. Mitochondrial swelling and the second-phase depolarization were inhibited by cyclosporin-A or the catalyst of $\mathrm{K}^{+} / \mathrm{H}^{+}$exchange nigericin, whereas the first-phase depolarization was not affected by either. In contrast, the methyl esters of FOSAA and $N$-EtFOSAA exhibited no depolarizing or MPT inducing activity. Results of this investigation demonstrate that the carboxylic acid moiety of the $N$-acetates is the active functional group, which triggers the MPT by perfluorinated sulfonamides.
\end{abstract}

\section{Introduction}

Since the early 1950s, $N$-alkyl perfluorooctane sulfonamides, including $N$-acetyl-perfluorooctanesufonamide (FOSAA) ${ }^{1}$ and $N$-ethylperfluorooctanesulfonamidoacetate ( $N$-EtFOSAA), have been widely used as surfactants on fabrics and papers, fire retardants, anticorrosion agents, and antistatic agents for film production, among other commercial applications (1). The perfluorooctane sulfonamides were manufactured for their chemical and thermal inertness and special surface-active characteristics. The high-energy fluorine-carbon bonds impart the physical and chemical stability that renders perfluorooctanes resistant to hydrolysis, photolysis, microbial degradation, and metabolism. Consequently, the perfluorooctanes are extremely persistent in the environment $(1,2)$.

The broad application, global distribution, and environmental persistence of these perfluorinated compounds has generated

* To whom correspondence should be addressed. Phone: (218) 7267927. Fax: (218) 726-8014. E-mail: obri0150@d.umn.edu.

$\dagger$ Department of Biochemistry and Molecular Biology, Toxicology Graduate Program, University of Minnesota, Medical School.

$\doteqdot$ Department of Chemistry, University of Minnesota.

$\S$ University of Coimbra.

${ }^{1}$ Abbreviations: ANOVA, analysis of variance; ANT, adenine nucleotide translocator; BSA, bovine serum albumin; CsA, cyclosporin A; DMSO, dimethysulfoxide; EGTA, ethylene glycol bis(2-aminoethyl ether)- $N, N, N^{\prime} N^{\prime}$ tetraacetic acid; FOSAA, perfluorooctanesulfonamidoacetate; GC-ECD, gas chromatography with electron capture detection; MPT, mitochondrial permeability transition; $N$-EtFOSAA, $N$-ethylperfluorooctanesulfonamidoacetate; PFOS, perfluorooctanesulfonate; $\mathrm{TPP}^{+}$, tetraphenylphosphonium ion; $\Delta \Psi$, mitochondrial membrane potential. considerable concern regarding their potential toxicity (3). The terminal degradation product of most $N$-alkyl perfluorooctane sulfonamides is perfluorooctanesulfonate (PFOS). PFOS and FOSAA have been identified in individual and pooled human serum samples from the United States general population (46 ), and it has been shown that FOSAA and $N$-EtFOSAA are metabolites in the biotransformation pathway of perfluorinated sulfonamido alcohols (7). PFOS itself has been detected in birds, fish, and mammalian tissues from the Canadian Artic, Baltic and Mediterranean Seas, and the Great Lakes of North America $(1,8-10)$. Although the source of PFOS in human blood is not well-characterized, it may likely involve exposure to PFOS itself or precursor molecules such as FOSAA and N-EtFOSAA (5). Because there is much yet to be learned about the distribution and degradation of perfluorooctane sulfonamides, estimating environmental exposure to any particular perfluorooctane including FOSAA and $N$-EtFOSAA is complicated and inexact.

There have been a number of studies with a variety of exposure regimens in which a wasting syndrome or cachexia has been observed in animals exposed to perfluorinated acids. The most prominent acute and subchronic high-dose effects of perfluorinated acids in adult rodents and monkeys are altered lipid metabolism, hepatocellular hypertrophy, and vacuolation, decreased serum cholesterol, decreased triglycerides, hepatomegaly, decreased body weight, and death (11-14). PFOS exposure has also been shown to have adverse developmental effects in rodents where in vitro exposure results not only in maternal weight loss but also in increased abortions, reduced 
birth weights, and a profound neonatal morbidity in rats (1517). In all of these studies the most prominent toxicological outcome appears to be a dose-dependent metabolic disruption. Inhibition of mitochondrial respiration and impairment of $\beta$-oxidation, as a result of uncoupling of oxidative phosphorylation or the induction of the mitochondrial permeability transition (MPT), have been implicated in a variety of pathologies related to disrupted lipid metabolism and hepatotoxicity (18). This suggests a potential role for mitochondrial dysfunction in the underlying mechanism of toxicity of the perfluorinated sulfonamides.

Multiple in vitro studies have shown the perfluorooctane sulfonamides to disrupt mitochondrial bioenergetics by three distinct mechanisms: uncoupling mitochondrial respiration, increasing nonspecific membrane permeability, and induction of calcium-dependent mitochondrial swelling (19-21). Specifically, FOSAA and $N$-EtFOSAA have been shown to be potent inducers of the MPT, which results in the release of cytochrome $c$, inhibition of respiration, and the generation of reactive oxygen species (22). The fact that these effects occur at low micromolar concentrations further supports the possibility that mitochondrial dysfunction may be an important factor in the pathogenesis caused by perfluorooctane sulfonamides.

The mitochondrial permeability transition represents a nonselective increase in the permeability of the inner mitochondrial membrane to solutes up to $1.5 \mathrm{kD}$ in size (23). It is characterized by dependence on matrix $\mathrm{Ca}^{2+}$ and is inhibited by elevated transmembrane potential, acidic matrix $\mathrm{pH}$, and the immunosuppressant cyclosporin-A (CsA) $(24-26) . \mathrm{Ca}^{2+}$ can itself induce the MPT, but most often the MPT is a result of $\mathrm{Ca}^{2+}$ acting in conjunction with various "inducing agents" such as fatty acids or oxidants (27). Associated with MPT induction is the rapid equilibration of solutes across the mitochondrial membranes leading to depolarization of membrane potential, osmotic swelling, inhibition of oxidative phosphorylation, and release of potential apoptogenic factors, (28) including cytochrome $c$ (29).

The purpose of the current investigation was to test the hypothesis that the $\mathrm{N}$-acetate moiety of FOSAA and $\mathrm{N}$ EtFOSAA is responsible for the induction of the MPT. To test this hypothesis, we synthesized methyl esters of the acetates and assessed them along with FOSAA and $N$-EtFOSAA for MPT inducing activity by measuring the change in $\Delta \Psi$ and mitochondrial swelling in the presence and absence of $\mathrm{Ca}^{2+}$, nigericin, and/or CsA. The results reported herein add significant new understanding to the mechanism by which $N$-acetyl perfluorooctane sulfonamides induce the MPT and identifies the $\mathrm{N}$-acetate as the critical functional group responsible for the activity of these compounds. The compounds chosen for inclusion in this investigation are depicted in Table 1.

\section{Experimental Procedures}

Mitochondrial Isolation. Mitochondria were isolated from liver of adult mixed-sex Sprague-Dawley rats (175-300 g body weight) by differential centrifugation (30). Rats were purchased from Harlan Sprague-Dawley (Madison, WI) and acclimated in an AAALACaccredited, climate-controlled animal-care facility for at least 3 days and fasted for $12-18 \mathrm{~h}$ prior to the experiment. Animals were euthanized in a $\mathrm{CO}_{2}$ chamber followed by decapitation. The liver was immediately excised, weighed, immersed in $40 \mathrm{~mL}$ of isolation medium (200 mM mannitol, $10 \mathrm{mM}$ sucrose, $5 \mathrm{mM}$ Hepes, $1 \mathrm{mM}$ EGTA, $\mathrm{pH}$ 7.4) and minced with scissors. The mitochondria were isolated and prepared as previously described $(21,22)$. The resulting mitochondrial pellet was re-suspended in a washing medium (200 $\mathrm{mM}$ mannitol, $10 \mathrm{mM}$ sucrose, $5 \mathrm{mM}$ Hepes, $\mathrm{pH}$ 7.4) to a final
Table 1. Structures of the $N$-Acetylperfluorooctanesulfonamides and Corresponding Esters

FOSAA Ester

protein concentration of $35-50 \mathrm{mg} / \mathrm{mL}$. Protein concentration was determined with the Bradford assay using BSA as a standard (31).

Diazomethane Preparation. $p$-Toluenesulfonylmethylnitrosamide (Diazald, Aldrich Chemical Co., 4.28 g., 2 mmol) in ether $(25 \mathrm{~mL})$ was added slowly through a nonground glass separation funnel to a solution of potassium hydroxide $(1.0 \mathrm{~g})$ in water $(2.0$ $\mathrm{mL}$ ) maintained at $65^{\circ} \mathrm{C}$ in a nonground glass distillation apparatus $(25 \mathrm{~mL})$. As the reaction proceeded, the diazomethane co-distilled with ether into a receiving flask containing ether $(10 \mathrm{~mL})$. When all the Diazald was added, additional ether $(10 \mathrm{~mL})$ was introduced and distillation continued until the distillate was colorless. The resulting yellow solution of diazomethane in ether was used without further purification.

Ester Preparation. The ethereal solution of diazomethane was introduced dropwise into a solution of the fluorinated acids dissolved in ether using a fire-polished disposable pipet. The addition of diazomethane was continued until no further nitrogen gas was evolved and the resulting ester solution remained a pale yellow color. The ether and excess diazomethane was allowed to evaporate in a hood and the residual esters used without further purification.

$$
\begin{aligned}
& \text { carboxylic acid }+ \text { diazomethane } \rightarrow \text { methyl ester }+ \text { nitrogen gas } \\
& \mathrm{R}-\mathrm{COOH} \quad \mathrm{CH}_{2} \mathrm{~N}_{2} \quad \mathrm{R}-\mathrm{COO}-\mathrm{CH}_{3} \quad \mathrm{iN}_{2}
\end{aligned}
$$

The authors chose the diazomethane esterification method because of its specificity. This method is a widely used analytical protocol for synthesizing and quantifying small quantities of highly valuable carboxylic acids where purity and yield are critical. The U.S. EPA recognizes diazomethane esterification as a preferred method for methyl derivatization of organic halo-acid herbicides for GC quantification (U.S. EPA Method 8151a Revision - 1, 1996). Urbansky et al. used diazomethane esterification to demonstrate the quantitation of concentrations of halocarboxylates near the lower detection limits of gas chromatography with electron capture detection (GC-ECD) $(32,33)$. Additionally, because analytical characterization of the perfluorinated test compounds is exceedingly 
challenging and cost-prohibitive, we chose the method in which we could have the highest confidence in the purity and yield of the product.

Mitochondrial Swelling. Changes in mitochondrial volume were estimated by changes in light scattering as monitored spectrophotometrically at $540 \mathrm{~nm}(21,34)$. Freshly isolated mitochondria were suspended at $0.5 \mathrm{mg} / \mathrm{mL}$ in $175 \mathrm{mM}$ sucrose, $5 \mathrm{mM}$ Hepes, 50 $\mathrm{mM} \mathrm{KCl}, 2.5 \mathrm{mM} \mathrm{KH}{ }_{2} \mathrm{PO}_{4}$, and $10 \mu \mathrm{M}$ EGTA (pH 7.4) supplemented with $2 \mu \mathrm{M}$ rotenone and $1 \mu \mathrm{g} / \mathrm{mL}$ oligomycin. The reaction was stirred continuously at $30{ }^{\circ} \mathrm{C}$ for $20 \mathrm{~min}$. The mitochondria were energized with $5 \mathrm{mM}$ succinate for 2 min before adding 25 $\mu \mathrm{M} \mathrm{CaCl}_{2}$. FOSAA, $N$-EtFOSAA, FOSAA methyl ester, or $N$-EtFOSAA methyl ester was added 2 min later. Where indicated, $2 \mu \mathrm{M}$ of the MPT inhibitor cyclosporin A (CsA) (24) was added just prior to succinate, or $200 \mathrm{nM}$ nigericin, a catalyst of $\mathrm{K}^{+} / \mathrm{H}^{+}$ exchange, was added just prior to the test compound. The criteria for establishing induction of the MPT was a decrease in light scattering of at least 0.5 absorbance units 6 min postaddition of test compound, which was inhibited completely by $2 \mu \mathrm{M}$ CsA. O'Brien and Wallace previously established the concentrations of FOSAA and $N$-EtFOSAA used in this study (22). The phase partitioning properties of the perfluorinated octanes suggest that these compounds do not partition to the aqueous or organic phase, but rather to a separate phase altogether (1). However, in vivo, the perfluorinated octanes with poor aqueous solubility, including $n$-ethyl perfluoroctanesulfonamido ethyl alcohol, tend to partition to the proteins in the serum and liver (35). With solubility in mind the concentration of the methyl esters of FOSAA and $N$-EtFOSAA tested were 10 times that of the corresponding acetates, and all experiments were run in a continuously mixed reaction chamber at $30^{\circ}$ to maximize ester partitioning to the mitochondrial fraction of the reaction.

TPP $^{+}$Methods. A $\mathrm{TPP}^{+}$selective electrode was prepared as described by $(36)$. Changes in membrane potential $(\Delta \Psi)$ of rat liver mitochondria were measured by a tetraphenylphosphonium ion $\left(\mathrm{TPP}^{+}\right)$sensitive electrode. Briefly, $0.5 \mathrm{mg}$ of protein $/ \mathrm{mL}$ was incubated at $30{ }^{\circ} \mathrm{C}$ in a buffered solution of $175 \mathrm{mM}$ sucrose, 50

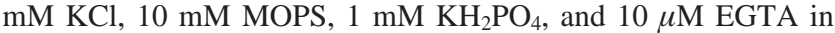
the presence of $2 \mu \mathrm{M} \mathrm{TPP}^{+}$, and supplemented with $2 \mu \mathrm{M}$ rotenone and $1 \mu \mathrm{g} / \mathrm{mL}$ oligomycin. The reaction was stirred continuously at $30{ }^{\circ} \mathrm{C}$ during the incubation. The mitochondria were energized with $5 \mathrm{mM}$ succinate for $1.5 \mathrm{~min}$ before adding $25 \mu \mathrm{M} \mathrm{CaCl}_{2}$. FOSAA, $N$-EtFOSAA, FOSAA methyl ester, $N$-EtFOSAA methyl ester, or a DMSO control was added 2 min later. Where indicated, $2 \mu \mathrm{M}$ of the MPT inhibitor cyclosporin A (CsA) or $200 \mathrm{nM}$ nigericin was added just prior to the test compound. As reported, phase 1 depolarization describes the change in $\Delta \Psi$ immediately upon addition of the test compound, and phase 2 depolarization describes the change in $\Delta \Psi 6 \mathrm{~min}$ following the addition of the test compound. Results were reported in millivolts $(\mathrm{mV})$, which were calculated as described (36-38). Through initial optimization we determined that $2 \mu \mathrm{M} \mathrm{TPP}{ }^{+}$provided the most sensitive and responsive measure of $\Delta \Psi$ for this study.

Reagents. All fluorochemical compounds were synthesized, characterized, and provided gratis by the 3M Company, St. Paul, MN. The methyl esters of the fluorochemical acetates were synthesized in house. Cyclosporin A was provided as a generous gift of Sandoz Pharmaceuticals (East Hanover, NJ, www.sandoz.com), Ultra Pure sucrose was purchased from ICN Biomedicals, Inc. (Aurora, $\mathrm{OH}$, www.icnbiomed.com), and all other reagents were from Sigma-Aldrich (St. Louis, MO, www.sigma-aldrich.com).

Hazardous Materials. Caution: Diazald is a severe skin irritant and all skin contact should be avoided. Diazomethane is extremely toxic, is an irritant, and has been cited as a carcinogen (39-41). Diazomethane has been known to explode unaccountably both as a gas and in solution. Rough surfaces are proven initiators of detonation (41). Extreme caution should be used when handling both.

Statistical Analysis. All experiments were repeated using freshly isolated hepatic mitochondria from at least three separate animals with a randomized selection of sexes. The results were analyzed

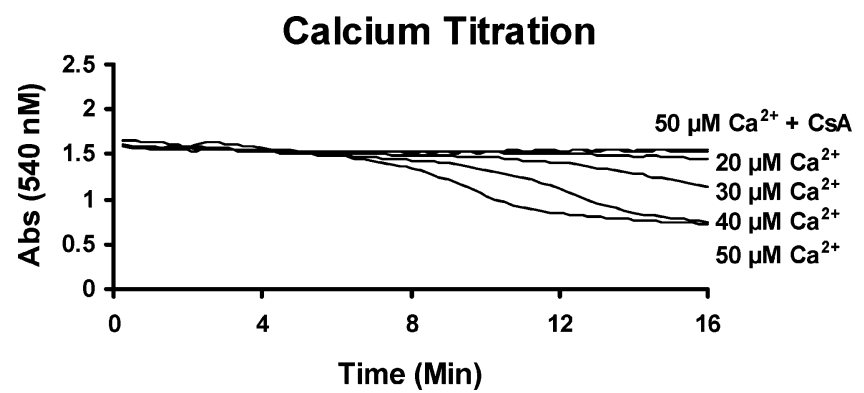

Figure 1. Calcium titration to measure permeability transition threshold. Swelling of rat liver mitochondria was monitored by light scattering at $540 \mathrm{~nm}$. $(0.5 \mathrm{mg}$ of protein $/ \mathrm{mL})$ were incubated at $30^{\circ} \mathrm{C}$ in a buffered solution of $175 \mathrm{mM}$ sucrose, $50 \mathrm{mM} \mathrm{KCl}, 10 \mathrm{mM}$ MOPS, $1 \mathrm{mM} \mathrm{KH}_{2} \mathrm{PO}_{4}, 10 \mu \mathrm{M}$ EGTA in the presence of $2 \mu \mathrm{M}$ rotenone, 5 $\mathrm{mM}$ succinate, $1 \mu \mathrm{g} / \mathrm{mL}$ oligomycin, plus $0,20,30,40$, and $50 \mu \mathrm{M}$ $\mathrm{Ca}^{2+}$, and $50 \mu \mathrm{M} \mathrm{Ca}^{2+}$ plus cyclosporin A.

by 2-way ANOVA and Tukey's post-hoc test. A probability of ( $p$ $<0.05$ ) was used as the criterion for statistical significance.

\section{Results}

Calcium Titration. $\mathrm{Ca}^{2+}$ is electrophoretically accumulated by mitochondria up to $1 \mu \mathrm{mol} / \mathrm{mg}$ of protein via the near 200 $\mathrm{mV}$ electronegative trans-membrane potential (42). However, there is a finite capacity which, if exceeded, triggers the mitochondria to undergo the induction of the mitochondrial permeability transition (MPT) $(23,43)$. In order to quantify calcium-loading capacity, which is considered to be a sensitive and specific indicator of mitochondrial competence and sensitivity to induction of the MPT (21), mitochondria were titrated with concentrations of calcium ranging from 0 to $100 \mathrm{nmol} / \mathrm{mg}$ of protein. Figure 1 is a representative tracing of a routine calcium titration. From this we established on average a concentration of $25 \mu \mathrm{M}$ calcium ( $50 \mathrm{nmol} / \mathrm{mg}$ of protein) as an amount that is below the calcium loading capacity of control mitochondria, yet sufficient to support induction of the MPT in response to selected inducing agents.

Mitochondrial Membrane Potential $(\Delta \Psi)$. Figures 2 and 3 illustrate a typical experiment for measuring the effects of the $N$-acetyl perfluorooctane sulfonamides (FOSAA and $N$ EtFOSAA, respectively) and the corresponding methyl esters on mitochondrial trans-membrane potential in the presence and absence of $\mathrm{Ca}^{2+}$, nigericin, and CsA. The results indicate that there are two phases of depolarization that occur upon addition of FOSAA and $N$-EtFOSAA. The initial, partial depolarization occurs immediately upon addition of $40 \mu \mathrm{M}$ FOSAA or $7 \mu \mathrm{M}$ $N$-EtFOSAA (Figures 2 and 3; panels B, C, D, and E) where there is approximately a $(+)(15-25) \mathrm{mV}$ change in $\Delta \Psi$. This event occurs in the presence or absence of $\mathrm{Ca}^{2+}$, nigericin, or CsA. In direct contrast, upon addition of the corresponding methyl esters of the acetates (Figures 2 and 3; panel F), there is no change in $\Delta \Psi$. Even at 10 times the concentration of the acetates, the esters still do not initiate depolarization or induce the MPT. The results of the initial depolarization for FOSAA, $N$-EtFOSAA in the presence and absence of $\mathrm{Ca}^{2+}$, nigericin, and CsA, and the corresponding methyl esters are presented quantitatively in Figure 4, phase 1 depolarization. The initial depolarization induced by the DMSO control and the esterified treatments is significantly less than that of the FOSAA or $N$-EtFOSAA treatments in both cases.

The slower phase of depolarization is reported as the change in $\Delta \Psi 6$ min after the addition of the test compound. In Figures 2 and 3, panel B illustrates the change in $\Delta \Psi$ by FOSAA and $N$-EtFOSAA in the presence of $\mathrm{Ca}^{2+}$ absent nigericin and CsA. 

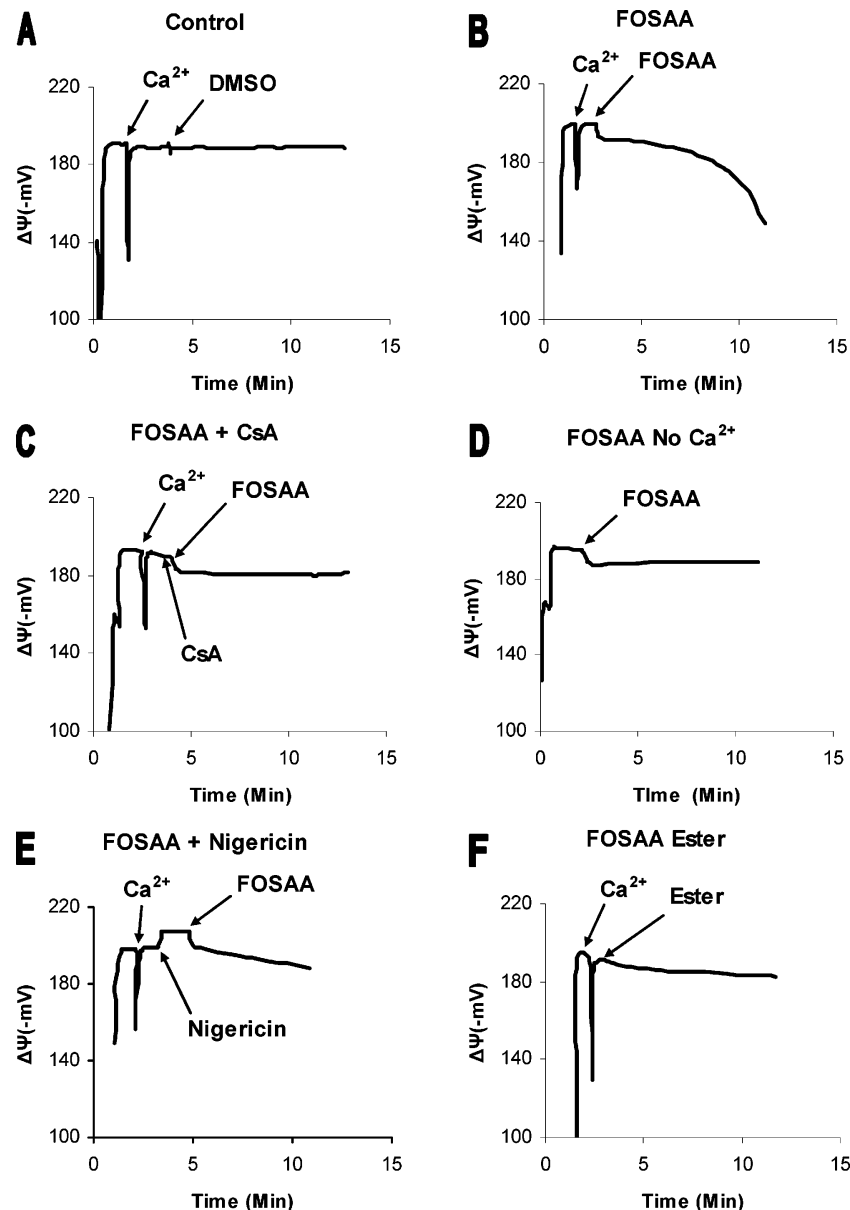

Figure 2. Membrane potential $(\Delta \Psi)$ of rat liver mitochondria was measured by a tetraphenylphosphonium ion $\left(\mathrm{TPP}^{+}\right)$sensitive electrode. Results reported in millivolts $(\mathrm{mV}) .0 .5 \mathrm{mg}$ of protein $/ \mathrm{mL}$ were incubated at $30{ }^{\circ} \mathrm{C}$ in a buffered solution of $175 \mathrm{mM}$ sucrose, $50 \mathrm{mM}$ $\mathrm{KCl}, 10 \mathrm{mM}$ MOPS, $1 \mathrm{mM} \mathrm{KH}_{2} \mathrm{PO}_{4}, 10 \mu \mathrm{M}$ EGTA in the presence of $2 \mu \mathrm{M} \mathrm{TPP}{ }^{+}, 2 \mu \mathrm{M}$ rotenone, $5 \mathrm{mM}$ succinate, and $1 \mu \mathrm{g} / \mathrm{mL}$ oligomycin: (A) $25 \mu \mathrm{M} \mathrm{Ca}^{2+}$, DMSO control (carrier); (B) $25 \mu \mathrm{M}$ $\mathrm{Ca}^{2+}, 40 \mu \mathrm{M}$ FOSAA; (C) $25 \mu \mathrm{M} \mathrm{Ca}^{2+}, 40 \mu \mathrm{M}$ FOSAA, plus $2 \mu \mathrm{M}$ cyclosporin A; (D) $40 \mu \mathrm{M}$ FOSAA absent of $\mathrm{Ca}^{2+}$; (E) $25 \mu \mathrm{M} \mathrm{Ca}^{2+}$, $40 \mu \mathrm{M}$ FOSAA, plus $200 \mathrm{nM}$ nigericin; and (F) $25 \mu \mathrm{M} \mathrm{Ca}^{2+}, 400 \mu \mathrm{M}$ FOSAA ester. Traces are representative of $3-5$ repetitions, each using separate mitochondrial preparations.

This secondary depolarization does not occur in the absence of $\mathrm{Ca}^{2+}$ (Figures 2 and 3; panel D) and is inhibited in the presence of either CsA or nigericin (Figures 2 and 3; panels $\mathrm{C}$ and $\mathrm{E}$, respectively). There is no occurrence of membrane depolarization in the presence of the DMSO control or the methyl esters of the acetates (Figures 2 and 3; panel F). The results of the secondary depolarization for FOSAA, $N$-EtFOSAA in the presence and absence of $\mathrm{Ca}^{2+}$, nigericin and $\mathrm{CsA}$, and the corresponding methyl esters are presented quantitatively in Figure 4, phase 2 depolarization. The secondary depolarization induced by the FOSAA or $N$-EtFOSAA $+\mathrm{Ca}^{2+}$ in both cases is significantly greater than that of the DMSO control. In all cases the absence of $\mathrm{Ca}^{2+}$, the presence of nigericin, $\mathrm{CsA}$, or the methyl esters of FOSAA and $N$-EtFOSAA significantly inhibited phase 2 depolarization caused by FOSAA and $N$ EtFOSAA plus $\mathrm{Ca}^{2+}$.

Mitochondrial Swelling. Calcium-dependent, CsA-inhibited mitochondrial swelling measured by light scattering at $540 \mathrm{nM}$ is a characteristic measure of MPT induction $(25,26)$. To verify that the observed membrane depolarization is related to the MPT induction, we recorded light scattering under the same treatment conditions as the membrane potential measurements. Figure 5
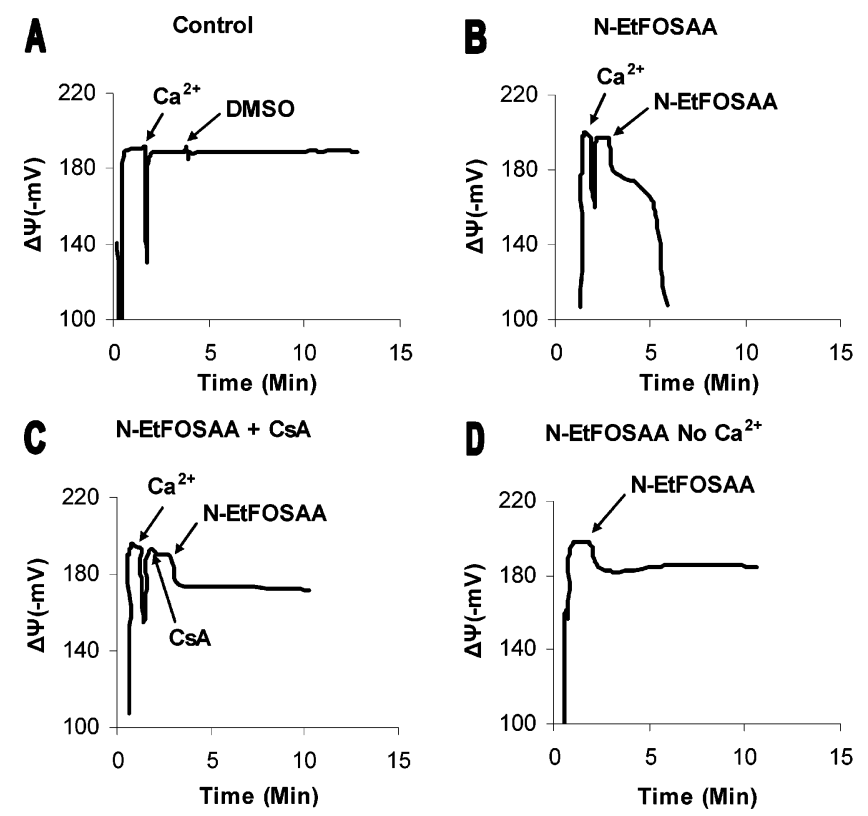

E N-EtFOSAA + Nigericin

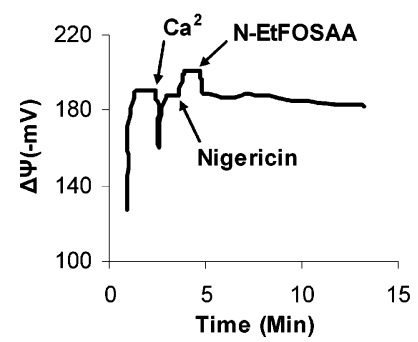

$F$

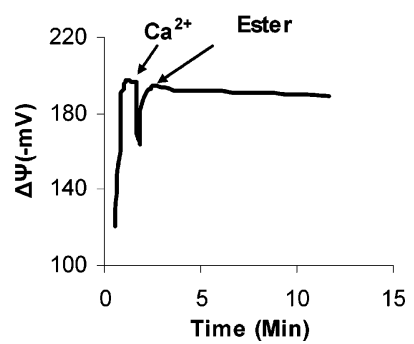

Figure 3. Membrane potential $(\Delta \Psi)$ of rat liver mitochondria was measured by a tetraphenylphosphonium ion $\left(\mathrm{TPP}^{+}\right)$sensitive electrode. Results reported in millivolts $(\mathrm{mV}) .0 .5 \mathrm{mg}$ of protein $/ \mathrm{mL}$ were incubated at $30^{\circ} \mathrm{C}$ in a buffered solution of $175 \mathrm{mM}$ sucrose, $50 \mathrm{mM}$

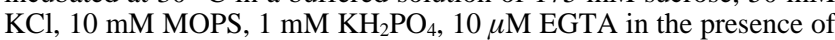
$2 \mu \mathrm{M} \mathrm{TPP}{ }^{+}, 2 \mu \mathrm{M}$ rotenone, $5 \mathrm{mM}$ succinate, and $1 \mu \mathrm{g} / \mathrm{mL}$ oligomycin: (A) $25 \mu \mathrm{M} \mathrm{Ca}^{2+}$, DMSO control (carrier); (B) $25 \mu \mathrm{M}$ $\mathrm{Ca}^{2+}, 7 \mu \mathrm{M} N$-EtFOSAA; (C) $25 \mu \mathrm{M} \mathrm{Ca}^{2+}, 7 \mu \mathrm{M} N$-EtFOSAA, plus 2 $\mu \mathrm{M}$ cyclosporin A; (D) $7 \mu \mathrm{M} \mathrm{N}$-EtFOSAA absent $\mathrm{Ca}^{2+}$; (E) $25 \mu \mathrm{M}$ $\mathrm{Ca}^{2+}, 7 \mu \mathrm{M} N$-EtFOSAA, plus $200 \mathrm{nM}$ nigericin; and (F) $25 \mu \mathrm{M} \mathrm{Ca}^{2+}$, $70 \mu \mathrm{M} N$-EtFOSAA ester. Traces are representative of $3-5$ repetitions, each using separate mitochondrial preparations.

illustrates the typical experimental run for testing the effects of the $\mathrm{N}$-acetyl perfluorooctane sulfonamides (FOSAA and $\mathrm{N}$ EtFOSAA) and the corresponding methyl ester test compounds on mitochondrial light scattering at $540 \mathrm{nM}$ in the presence and absence of nigericin or CsA. Figure 5, panels A and B, show that $40 \mu \mathrm{M}$ FOSAA or $7 \mu \mathrm{M} N$-EtFOSAA induced mitochondrial swelling, both of which are inhibited by CsA. In contrast, $400 \mu \mathrm{M}$ FOSAA methyl ester (Figure 5; panel A) and $70 \mu \mathrm{M}$ $\mathrm{N}$-EtFOSAA methyl ester do not induce mitochondrial swelling (Figure 5; panel B). Figure 5 panel $\mathrm{C}$ depicts nigericin inhibition of mitochondrial swelling. Two hundred nanomolar nigericin in a buffer containing $51 \mathrm{mM} \mathrm{K}^{+}$protects against mitochondrial swelling induced by FOSAA and $N$-EtFOSAA. In all cases there is a delay from the time of test compound addition until the initiation of mitochondrial swelling.

The results of mitochondrial swelling induced by FOSAA, $N$-EtFOSAA in the presence of $\mathrm{Ca}^{2+}$, plus or minus nigericin or CsA, and the corresponding methyl esters are presented quantitatively in Figure 5, panel D. In each case there is a significant decrease in light scattering by FOSAA and $\mathrm{N}$ EtFOSAA versus control, whereas the absence of $\mathrm{Ca}^{2+}$ or in the presence of nigericin or CsA protects against mitochondrial 

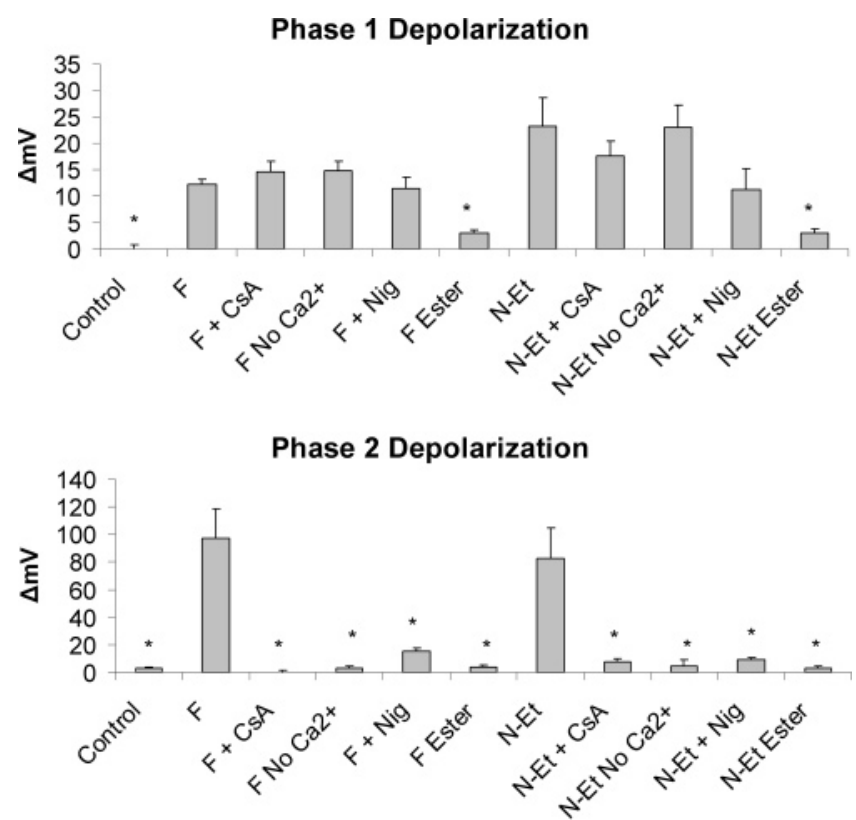

Figure 4. $\mathrm{F}$ (FOSAA) and $N$-Et ( $N$-EtFOSAA) induced change in membrane potential $(\Delta \Psi)$ as measured by a $\left(\mathrm{TPP}^{+}\right)$sensitive electrode. Results reported in millivolts $(\mathrm{mV})$. Phase 1 depolarization is the immediate change in $\Delta \Psi$ upon addition of the test compound. Phase 2 depolarization is the change in $\Delta \Psi 6$ min after the addition of the test compound. The concentrations and additions are as reported in Figures 2 and 3. Bars represent the mean \pm S.E.M. of 3-5 repetitions each analyzed by 2-way ANOVA and Tukey's post-hoc test. A probability of $(p<0.05)$ was used as the criterion for statistical significance. $\left(^{*}\right)$ Statistically significant difference compared to treatment alone.

swelling. The change in light scattering caused by the methyl esters was significantly less than that induced by FOSAA and $N$-EtFOSAA plus $\mathrm{Ca}^{2+}$. This data indicates that swelling is directly related to induction of the MPT by the $N$-acetyl perfluorooctane sulfonamides and that the $N$-acetate of FOSAA and $N$-EtFOSAA is the critical functional group responsible for the activity of these compounds.

\section{Discussion}

The results of this investigation demonstrate that induction of the MPT by the $N$-acetyl perfluorooctane sulfonamides, FOSAA and N-EtFOSAA, is an event that is entirely dependent upon the carboxylic acid functional group. Induction of the MPT is preceded by a partial $(15-25 \mathrm{mV})$ depolarization of $\Delta \Psi$ that occurs immediately upon addition of the $\mathrm{N}$-acetyl test compounds (Figures 4; phase 1 depolarization). This initial depolarization occurs in the absence of $\mathrm{Ca}^{2+}$ and in the presence of cyclosporin-A, indicating that it is not a result of MPT induction. The fact that the initial depolarization is completely blocked by methyl-esterification of the acids, but not by nigericin, is evidence that the effect requires the carboxyl moiety and appears to be the result of an immediate translocation of charge across the inner mitochondrial membrane. This electrophoretic interpretation of the $\mathrm{TPP}^{+}$data is supported by the fact that in the presence of CsA there is no swelling (Figure 5), whereas nonspecific swelling would be expected if the initial depolarization were the result of generalized membrane dissolution by the perfluorinated sulfonamides.

The second component of membrane depolarization is the gradual and complete collapse of $\Delta \Psi$ (Figures 2 and 3, panel B). This secondary phase requires $\mathrm{Ca}^{2+}$ and is inhibited by CsA or nigericin, indicating that it is a consequence of MPT induction. The secondary-phase depolarization does not occur with the corresponding methyl esters of the acetates, providing further evidence that mitochondrial membrane depolarization and induction of the MPT are entirely dependent upon the presence of the carboxylic acid.

The methyl esters of the acetates used in this investigation do not induce mitochondrial swelling (Figure 5A,B), nor do they cause membrane depolarization as is observed with FOSAA and $N$-EtFOSAA. Membrane integrity and membrane potential are not compromised in the presence of the esters; therefore, general membrane solubility of the perfluorinated compounds is likely not the disruptive mechanism observed in this investigation. These findings are in agreement with those of Starkov and Wallace who found that the perfluorinated compounds without amide or acidic moieties do not disrupt mitochondrial bioenergetics (21). Under every treatment condition involving FOSAA and $N$-EtFOSAA the initial depolarization occurs immediately upon addition, yet only in the presence of $\mathrm{Ca}^{2+}$ does the secondary depolarization occur. This evidence suggests a specific interaction with mitochondrial targets leading to an immediate depolarization of membrane potential, which does not occur in the absence of the carboxylic acid moiety.

The relationship between mitochondrial depolarization and MPT induction has been the subject of many studies. It has been demonstrated that at low membrane potential mitochondrial redox potential is decreased because $\mathrm{NAD}(\mathrm{P}) \mathrm{H}$ transhydrogenase cannot sustain adequate NADPH and GSH levels (44), which may result in MPT induction. In fact, Castilho et al. proposed that a decrease in $\Delta \Psi$ results in $\mathrm{Ca}^{2+}$-induced ROS accumulation, thiol cross-linking, and MPT induction (45). Others advocate that the MPT is voltage-sensitive and a decrease in $\Delta \Psi$ results in a greater likelihood of MPT induction $(25,46)$. Regardless of the initiating mechanism, the final outcome of MPT induction is a complete collapse of the $\Delta \Psi$ and, consequently, the electrochemical proton gradient (47).

There have been a multitude of studies regarding the mechanism by which fatty acids induce the MPT. It has been reported that fatty acid induced MPT is a result of proton translocation across the inner mitochondrial membrane which lowers $\Delta \Psi$ to a MPT gating potential $(27,48)$. Once the gating potential is achieved, MPT induction is more likely. Other proposed mechanisms include the cycling of monovalent cations other than protons caused by fatty acid membrane translocation, again resulting in an electrogenic triggering of mitochondrial swelling (49). These mechanisms appear to require the ionized fatty acid be shuttled back to the inner membrane space through a process that apparently involves transporters such as the adenine nucleotide translocator (ANT) (50) or dicarboxylate carrier $(51,52)$. Regardless of the specific target, each proposed mechanism has in common a process by which fatty acids "flipflop" back and forth across the inner mitochondrial membrane and cause a dissipation of $\Delta \Psi$. Although the perfluorinated carbon tail of FOSAA and $N$-EtFOSAA imparts a more rigid physical character to the molecule, the aliphatic head of the sulfonamide will have properties comparable to non-fluorinated fatty acids. It is possible that the $N$-acetyl perfluorinated sulfonamides interact with the inner mitochondrial membrane in a fashion similar to non-fluorinated fatty acids.

The findings of this investigation allow us to propose that the initial depolarization of mitochondrial $\Delta \Psi$ is an electrophoretic event caused by the $N$-acetyl perfluorooctane sulfonamides translocating charge, most likely protons, across the mitochondrial membranes. The specific mechanism of the observed charge translocation is not completely clear. It may 
FOSAA vs Ester

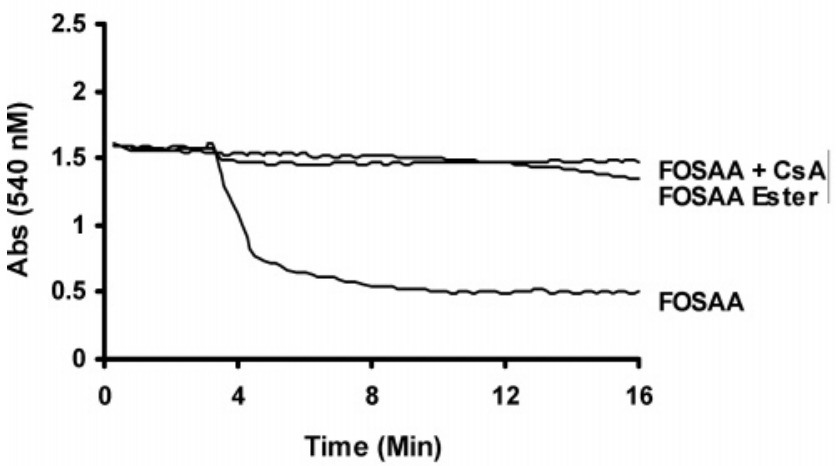

Nigericin Inhibition of Swelling

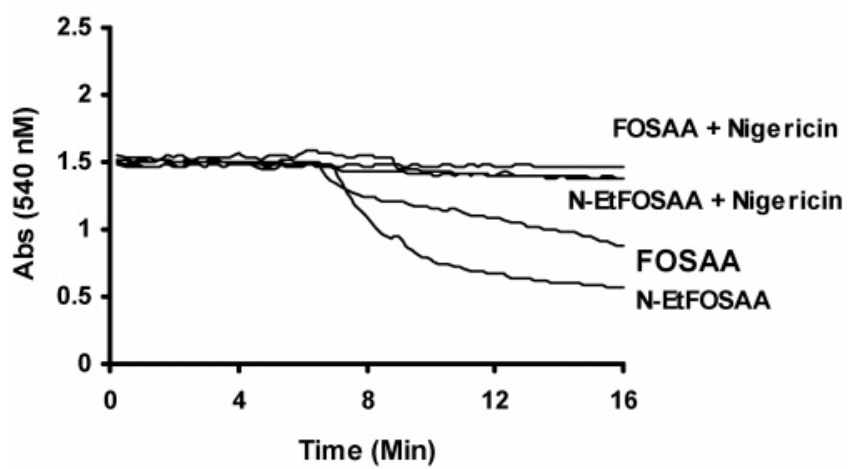

N-EtFOSAA vs Ester

B

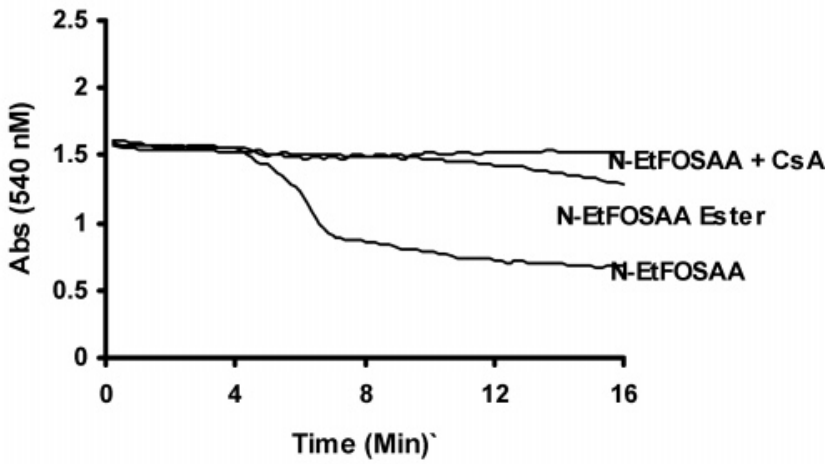

Change in Abs (6 Minutes Post

D

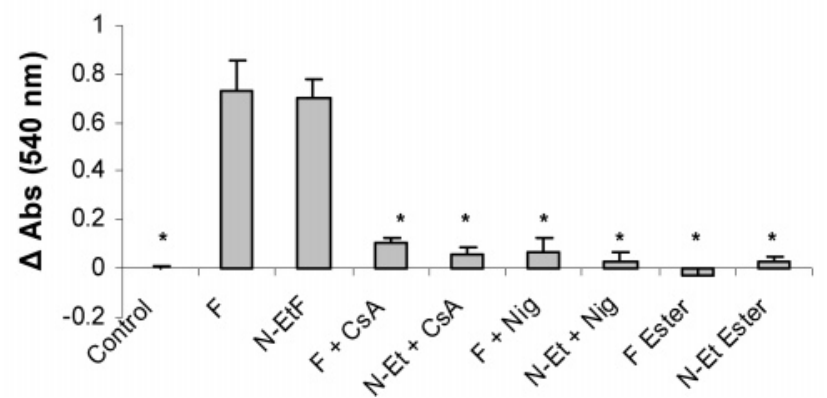

Figure 5. Swelling of rat liver mitochondria was monitored by light scattering at $540 \mathrm{~nm} .\left(0.5 \mathrm{mg}\right.$ of protein/mL) were incubated at $30{ }^{\circ} \mathrm{C}$ in a

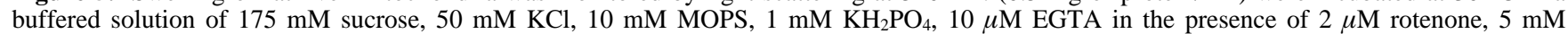
succinate, $1 \mu \mathrm{g} / \mathrm{mL}$ oligomycin, $25 \mu \mathrm{M} \mathrm{Ca}^{2+}$ plus the selected perfluorinated compound or DMSO (carrier) at the indicated concentration in the presence and absence of $2 \mu \mathrm{M}$ cyclosporin A (CsA). Controls were treated with $25 \mu \mathrm{M} \mathrm{Ca}^{2+}$. Mitochondrial swelling traces are representative of 3 repetitions, each using separate mitochondrial preparations. (A) $400 \mu \mathrm{M}$ FOSAA \pm cyclosporin A, $400 \mathrm{M}$ FOSAA ester; (B) $7 \mu \mathrm{M} N$-Et-FOSAA \pm cyclosporin A, $70 \mu \mathrm{M} \mathrm{N}$-EtFOSAA ester; (C) $7 \mu \mathrm{M} N$-EtFOSAA, $40 \mu \mathrm{M}$ FOSAA in the presence and absence of $200 \mathrm{nM}$ nigericin; (D) F (FOSAA) and $N$-Et ( $N$-EtFOSAA) induced decrease in absorbance measured at $540 \mathrm{nM}$. Results reported as change in arbitrary absorbance units 6 min after the addition of the test compound. Bars represent the mean \pm S.E.M. of 3 repetitions each analyzed by 2-way ANOVA and Tukey's post-hoc test. A probability of $(p<0.05)$ was used as the criterion for statistical significance. $\left.{ }^{*}\right)$ Statistically significant difference compared to the treatment.

be the result of a rearrangement of membrane phospholipids, which induces a generalized proton leak, or it could be due to direct protonophoric activity of FOSAA and $N$-EtFOSAA. Calculated $\mathrm{pK}_{\mathrm{a}}$ values of less than 5 for the test compounds would support the first interpretation, but the fact that the esters do not induce phase 1 depolarization, or that there is no mitochondrial swelling induced by FOSAA or $N$-EtFOSAA in the presence of CsA, supports the protonophoric interpretation. In either case, as a consequence of phase 1 depolarization, $\Delta \Psi$ is depressed, causing the mitochondria to become more susceptible to MPT induction. The hyperpolarization and matrix acidification induced by the presence of nigericin $(25,53)$, which inhibits the MPT, also supports this interpretation. The secondary membrane depolarization observed is specifically a consequence of MPT induction. The results also provide insight as to why the fully substituted sulfonamide $N$-EtFOSAA is approximately 5 times more potent than the secondary substituted sulfonamide FOSAA. It is estimated that the $\mathrm{pK}_{\mathrm{a}}$ of the proton on the secondary substituted sulfonamide is quite low due to its proximity to the strong electron-withdrawing effects of fluorine, and therefore, the secondary amide of FOSAA would be present in its anionic state under the conditions of these experiments. This anionic state would render FOSAA less soluble in the mitochondrial membranes, whereas the fully substituted sulfonamide $N$-EtFOSAA does not have that anionic site and therefore is suspected to be much more membrane-soluble.

In conclusion, the fact that the pathologies expressed in vivo resulting from exposure to perfluorinated acids in rodents and monkeys are altered lipid metabolism, hepatocellular hypertrophy and vacuolation, decreased serum cholesterol, decreased triglycerides, hepatomegaly, and decreased body weight with no obvious histopathology provides strong evidence that these events are metabolic in nature. PFOS has generated considerable interest because of its environmental penetrance and persistence. It has been detected in a variety of species of wildlife worldwide with mean liver concentrations as high as $1.7 \mathrm{ppm}$ in predators such as polar bears (9). Exposure of the general human population is demonstrated by the detection of PFOS in banked serum at concentrations of $0.03-0.175 \mathrm{ppm}(4,6,54)$ with occupational exposures of fluorochemical production workers reported at $0.04-12.70 \mathrm{ppm}$ serum PFOS (55). Since PFOS has been suggested to be the terminal metabolite of the $\mathrm{N}$-alkylperfluorooctanesulfonamides in vivo $(3,14,17)$, the detection of PFOS in human or environmental monitoring studies may reflect either direct exposure to PFOS itself or exposures to more complex structural analogs. The concentrations used in this study are higher than those currently observed in animal or human serum samples (FOSAA, approximately $22 \mathrm{ppm}$, and $\mathrm{N}$ EtFOSAA, approximately $3.2 \mathrm{ppm}$ compared to the PFOS concentration of $12 \mathrm{ppm}$ in humans). However, the in vivo partitioning of the perfluorinated sulfonamides to proteins in the liver and serum, and the fact that FOSAA and $N$-EtFOSAA induce acute in vitro outcomes resulting in a distinct disruption of mitochondrial bioenergetics that may be related to the general metabolic disruption observed in vivo, supports the relevance of the concentrations applied. More importantly, the concentra- 
tions of PFOS observed in serum and liver of neonates with high mortality in a PFOS toxicity study (56) exceed the concentrations of $N$-EtFOSAA used in this study by more than 10-fold. Finally, identification of the carboxylic acid moiety as requisite to initiate the MPT adds significant new understanding to the process by which $N$-acetyl perfluorooctane sulfonamides disrupt mitochondrial bioenergetics and supports the hypothesis that this disruption plays a significant role in the observed pathological outcomes in vivo.

Acknowledgment. This work was supported by a generous grant from the 3M Company. The authors would like to thank James A. Bjork, LaRae Peterson, and Kaleb C. Lund for their technical support and insight, and Dr. Antonio J. Moreno of Coimbra University, Coimbra, Portugal, for preparation of the custom-made $\mathrm{TPP}^{+} \mathrm{Cl}^{-}$electrode. The authors would also like to thank Vikki Swenson for her dedicated work on the TOC graphic. Paulo J. Oliveira is supported by a grant from the Portuguese Foundation for Science and Technology (SFRH/ BPD/8359/2002).

\section{References}

(1) Giesy, J. P., and Kannan, K. (2002) Perfluorochemical surfactants in the environment. Environ. Sci. Technol. 36, 146A-152A.

(2) Shabalina, I. G., Panaretakis, T., Bergstrand, A., and DePierre, J. W. (1999) Effects of the rodent peroxisome proliferator and hepatocarcinogen, perfluorooctanoic acid, on apoptosis in human hepatoma HepG2 cells. Carcinogenesis 20, 2237-2246.

(3) Renner, R. (2001) Growing concern over perfluorinated chemicals Environ. Sci. Technol. 35, 154A-160A.

(4) Hansen, K. J., Clemen, L. A., Ellefson, M. E., and Johnson, H. O. (2001) Compound-specific, quantitative characterization of organic fluorochemicals in biological matrices. Environ. Sci. Technol. 35, 766770.

(5) Olsen, G. W., Church, T. R., Miller, J. P., Burris, J. M., Hansen, K. J., Lundberg, J. K., Armitage, J. B., Herron, R. M., Medhdizadehkashi, Z., Nobiletti, J. B., O'Neill, E. M., Mandel, J. H., and Zobel, L. R. (2003) Perfluorooctanesulfonate and other fluorochemicals in the serum of American Red Cross adult blood donors. Environ. Health Perspect $111,1892-1901$.

(6) Olsen, G. W., Church, T. R., Larson, E. B., van Belle, G., Lundberg, J. K., Hansen, K. J., Burris, J. M., Mandel, J. H., and Zobel, L. R. (2004) Serum concentrations of perfluorooctanesulfonate and other fluorochemicals in an elderly population from Seattle, Washington. Chemosphere 54, 1599-1611.

(7) Xu, L., Krenitsky, D. M., Seacat, A. M., Butenhoff, J. L., and Anders, M. W. (2004) Biotransformation of N-ethyl-N-(2-hydroxyethyl)perfluorooctanesulfonamide by rat liver microsomes, cytosol, and slices and by expressed rat and human cytochromes P450. Chem. Res. Toxicol. 17, 767-775.

(8) Kannan, K., Corsolini, S., Falandysz, J., Oehme, G., Focardi, S., and Giesy, J. P. (2002) Perfluorooctanesulfonate and related fluorinated hydrocarbons in marine mammals, fishes, and birds from coasts of the Baltic and the Mediterranean Seas. Environ. Sci. Technol. 36, $3210-3216$

(9) Martin, J. W., Smithwick, M. M., Braune, B. M., Hoekstra, P. F., Muir, D. C., and Mabury, S. A. (2004) Identification of long-chain perfluorinated acids in biota from the Canadian Arctic. Environ. Sci. Technol. 38, 373-380.

(10) Key, B. D., Howell, R. D., and Criddle, C. S. (1998) Defluorination of Organofluorine Sulfur Compounds by Pseudomonas Sp. Strain D2. Environ. Sci. Technol. 32, 2283-2287.

(11) Vanden Heuvel, J. P., Kuslikis, B. I., Van Rafelghem, M. J., and Peterson, R. E. (1991) Tissue distribution, metabolism, and elimination of perfluorooctanoic acid in male and female rats. J. Biochem. Toxicol. 6, 83-92.

(12) Van Rafelghem, M. J., Mattie, D. R., Bruner, R. H., and Andersen, M. E. (1987) Pathological and hepatic ultrastructural effects of a single dose of perfluoro-n-decanoic acid in the rat, hamster, mouse, and guinea pig. Fundam. Appl. Toxicol. 9, 522-540.

(13) Goldenthal, E. I., Jessup, D. C., Geil, R. G., and Mehring, J. S. (1978b), Ninety-day subacute rhesus monkey toxicity study. Study No. 137092, International Research and Development Corporation, Mattawan, MI (U.S. EPA Docket No. 8(e)HQ-1180-00374).

(14) Seacat, A. M., Thomford, P. J., Hansen, K. J., Olsen, G. W., Case, M. T., and Butenhoff, J. L. (2002) Subchronic toxicity studies on perfluorooctanesulfonate potassium salt in cynomolgus monkeys. Toxicol. Sci. 68, 249-264.
(15) Case, M. T., York, R. G., and Christian, M. S. (2001) Rat and rabbit oral developmental toxicology studies with two perfluorinated compounds. Int. J. Toxicol. 20, 101-109.

(16) Luebker, D. J., York, R. G., Hansen, K. J., Moore, J. A., and Butenhoff, J. L. (2005) Neonatal mortality from in utero exposure to perfluorooctanesulfonate (PFOS) in Sprague-Dawley rats: dose-response, and biochemical and pharamacokinetic parameters. Toxicology 215, 149169.

(17) Thibodeaux, J. R., Hanson, R. G., Rogers, J. M., Grey, B. E., Barbee, B. D., Richards, J. H., Butenhoff, J. L., Stevenson, L. A., and Lau, C. (2003) Exposure to perfluorooctane sulfonate during pregnancy in rat and mouse. I: maternal and prenatal evaluations. Toxicol. Sci. 74 , 369-381.

(18) Jaeschke, H., Gores, G. J., Cederbaum, A. I., Hinson, J. A., Pessayre, D., and Lemasters, J. J. (2002) Mechanisms of hepatotoxicity. Toxicol. Sci. $65,166-176$.

(19) Panaretakis, T., Shabalina, I. G., Grander, D., Shoshan, M. C., and DePierre, J. W. (2001) Reactive oxygen species and mitochondria mediate the induction of apoptosis in human hepatoma HepG2 cells by the rodent peroxisome proliferator and hepatocarcinogen, perfluorooctanoic acid. Toxicol. Appl. Pharmacol. 173, 56-64.

(20) Keller, B. J., Marsman, D. S., Popp, J. A., and Thurman, R. G. (1992) Several nongenotoxic carcinogens uncouple mitochondrial oxidative phosphorylation. Biochim. Biophys. Acta 1102, 237-244.

(21) Starkov, A. A., and Wallace, K. B. (2002) Structural determinants of fluorochemical-induced mitochondrial dysfunction. Toxicol. Sci. 66, 244-252.

(22) O'Brien, T. M., and Wallace, K. B. (2004) Mitochondrial permeability transition as the critical target of $\mathrm{N}$-acetyl perfluorooctane sulfonamide toxicity in vitro. Toxicol. Sci. 82, 333-340.

(23) Bernardi, P. (1996) The permeability transition pore. Control points of a cyclosporin A-sensitive mitochondrial channel involved in cell death. Biochim. Biophys. Acta 1275, 5-9.

(24) Broekemeier, K. M., Dempsey, M. E., and Pfeiffer, D. R. (1989) Cyclosporin A is a potent inhibitor of the inner membrane permeability transition in liver mitochondria. J. Biol. Chem. 264, 7826-7830.

(25) Bernardi, P., Vassanelli, S., Veronese, P., Colonna, R., Szabo, I., and Zoratti, M. (1992) Modulation of the mitochondrial permeability transition pore. Effect of protons and divalent cations. J. Biol. Chem 267, 2934-2939.

(26) Haworth, R. A., and Hunter, D. R. (1979) The $\mathrm{Ca}^{2+}$-induced membrane transition in mitochondria. II. Nature of the $\mathrm{Ca}^{2+}$ trigger site. Arch. Biochem. Biophys. 195, 460-467.

(27) Petronilli, V., Nicolli, A., Costantini, P., Colonna, R., and Bernardi, P. (1994) Regulation of the permeability transition pore, a voltagedependent mitochondrial channel inhibited by cyclosporin A. Biochim. Biophys. Acta 1187, 255-259.

(28) Kroemer, G., and Reed, J. C. (2000) Mitochondrial control of cell death. Nat. Med. 6, 513-519.

(29) Cai, J., Yang, J., and Jones, D. P. (1998) Mitochondrial control of apoptosis: the role of cytochrome c. Biochim. Biophys. Acta 1366 , $139-149$.

(30) Zhou, S., and Wallace, K. B. (1999) The effect of peroxisome proliferators on mitochondrial bioenergetics. Toxicol. Sci. 48, 8289.

(31) Bradford, M. M. (1976) A Rapid and Sensitive Method for the Quantitation of Microgram Quantities of Protein Utilizing the Principle of Protein-Dye Binding. Anal. Biochem. 72, 248-254.

(32) Urbansky, E. T., and Bashe, W. J. (2000) Comparative methodology in the determination of [alpha]-oxocarboxylates in aqueous solution Ion chromatography versus gas chromatography after oximation, extraction and esterification. J. Chromatogr. A 867, 143-149.

(33) Urbansky, E. T., Freeman, D. M., and Rubios, F. J. (2000) Ascorbic acid reduction of residual active chlorine in potable water prior to halocarboxylate determination. J. Environ. Monit. 2, 253-256.

(34) Henry, T. R., and Wallace, K. B. (1995) Differential mechanisms of induction of the mitochondrial permeability transition by quinones of varying chemical reactivities. Toxicol. Appl. Pharmacol. 134, 195203.

(35) Luebker, D. J., Hansen, K. J., Bass, N. M., Butenhoff, J. L., and Seacat, A. M. (2002) Interactions of fluorochemicals with rat liver fatty acidbinding protein. Toxicology 176, 175-185.

(36) Kamo, N., Muratsugu, M., Hongoh, R., and Kobatake, Y. (1979) Membrane potential of mitochondria measured with an electrode sensitive to tetraphenyl phosphonium and relationship between proton electrochemical potential and phosphorylation potential in steady state. J. Membr. Biol. 49, 105-121.

(37) Drachev, L. A., Kondrashin, A. A., Samuilov, V. D., and Skulachev, V. P. (1975) Generation of electric potential by reaction center complexes from Rhodospirillum rubrum. FEBS Lett. 50, 219-222.

(38) Muratsugu, M., Kamo, N., Kurihara, K., and Kobatake, Y. (1977) Selective electrode for dibenzyl dimethyl ammonium cation as 
indicator of the membrane potential in biological systems. Biochim. Biophys. Acta 464, 613-619.

(39) Gutsche, C. D. (1954) Organic Reactions, Vol. VIII, John Wiley and Sons, New York.

(40) De Boer, T. J., and Backer, H. J. (1963) Organic Synthesis, Collective Volume IV, John Wiley \& Sons, New York.

(41) Schoental, R. (1960) Carcinogenic Action of Diazomethane and of Nitroso-N-methyl Urethane. Nature 188, 420-421.

(42) Gunter, K. K., and Gunter, T. E. (1994) Transport of calcium by mitochondria. J. Bioenerg. Biomembr. 26, 471-485.

(43) Zoratti, M., and Szabo, I. (1995) The mitochondrial permeability transition. Biochim. Biophys. Acta. 1241, 139-176.

(44) Vercesi, A. E. (1987) The participation of NADP, the transmembrane potential and the energy-linked $\operatorname{NAD}(\mathrm{P})$ transhydrogenase in the process of $\mathrm{Ca}^{2+}$ efflux from rat liver mitochondria. Arch. Biochem. Biophys. 252, 171-178.

(45) Castilho, R. F., Kowaltowski, A. J., Meinicke, A. R., Bechara, E. J., and Vercesi, A. E. (1995) Permeabilization of the inner mitochondria membrane by $\mathrm{Ca}^{2+}$ ions is stimulated by t-butyl hydroperoxide and mediated by reactive oxygen species generated by mitochondria. Free Radic. Biol. Med. 18, 479-486.

(46) Petronilli, V., Costantini, P., Scorrano, L., Colonna, R., Passamonti, S., and Bernardi, P. (1994) The voltage sensor of the mitochondrial permeability transition pore is tuned by the oxidation-reduction state of vicinal thiols. Increase of the gating potential by oxidants and its reversal by reducing agents. J. Biol. Chem. 269, 16638-16642.

(47) Dalla Via, L., Di Noto, V., and Toninello, A. (1998) Spermine binding to liver mitochondria deenergized by ruthenium red plus either FCCP or antimycin A. FEBS Lett. 422, 36-42.

(48) Bernardi, P., Penzo, D., and Wojtczak, L. (2002) Mitochondrial energy dissipation by fatty acids. Mechanisms and implications for cell death. Vitam. Horm. 65, 97-126.
(49) Schonfeld, P., Wieckowski, M. R., and Wojtczak, L. (2000) Longchain fatty acid-promoted swelling of mitochondria: further evidence for the protonophoric effect of fatty acids in the inner mitochondrial membrane. FEBS Lett. 471, 108-112.

(50) Skulachev, V. P. (1991) Fatty acid circuit as a physiological mechanism of uncoupling of oxidative phosphorylation. FEBS Lett. 294, 158162.

(51) Schonfeld, P., Wieckowski, M. R., and Wojtczak, L. (1997) Thyroid hormone-induced expression of the ADP/ATP carrier and its effect on fatty acid-induced uncoupling of oxidative phosphorylation. FEBS Lett. 416, 19-22.

(52) Wieckowski, M. R., and Wojtczak, L. (1997) Involvement of the dicarboxylate carrier in the protonophoric action of long-chain fatty acids in mitochondria. Biochem. Biophys. Res. Commun. 232, 414417.

(53) Reed, P. W. (1979) Ionophores. Methods Enzymol. 55, 435-454.

(54) Olsen, G. W., Hansen, K. J., Stevenson, L. A., Burris, J. M., and Mandel, J. H. (2003) Human donor liver and serum concentrations of perfluorooctanesulfonate and other perfluorochemicals. Environ. Sci. Technol. 37, 888-891.

(55) Olsen, G. W., Burris, J. M., Burlew, M. M., and Mandel, J. H. (2003) Epidemiologic assessment of worker serum perfluorooctanesulfonate (PFOS) and perfluorooctanoate (PFOA) concentrations and medical surveillance examinations. J. Occup. Environ. Med. 45, 260-270.

(56) Lau, C., Thibodeaux, J. R., Hanson, R. G., Rogers, J. M., Grey, B. E., Stanton, M. E., Butenhoff, J. L., and Stevenson, L. A. (2003) Exposure to perfluorooctane sulfonate during pregnancy in rat and mouse. II: postnatal evaluation. Toxicol. Sci. 74, 382-392.

TX060132R 